\title{
LIMIT BEHAVIOR OF SOLUTIONS OF STOCHASTIC DIFFERENTIAL EQUATIONS
}

BY

\section{AVNER FRIEDMAN( 1 )}

ABSTRACT. Consider a system of $m$ stochastic differential equations $d \xi=a(t, \xi) d t+\sigma(t, \xi) d w$. The limit behavior of $\xi(t)$, as $t \rightarrow \infty$, is studied. Estimates of the form $E|\xi(t)-\bar{\sigma} w(t)|^{2}=O\left(t^{1-\delta}\right)$ are derived, and various applications are given.

Introduction. Consider a system of $m$ stochastic differential equations

$$
d \xi(t)=a(t, \xi(t)) d t+\sigma(t, \xi(t)) d w(t)
$$

where $a=\left(a_{1}, \cdots, a_{m}\right), \sigma$ is an $m \times m$ matrix $\left(\sigma_{i j}\right), w(t)=\left(w_{1}(t), \cdots, w_{m}(t)\right)$, and the $w_{i}(t)$ are independent Brownian motions. The fundamental theory for such a system is presented in detail in [5], [6] (see also [2], [12]). In this paper we are concerned with the behavior of solutions $\xi(t)$ as $t \rightarrow \infty$. Our purpose is to find general conditions on $a$ and $\sigma$ under which the solutions will have asymptotic behavior similar to that of a Brownian motion. Some results in this direction were obtained by Kulinič ([7]-[10]) and in Gihman-Skorohod [6]. These results, however, are derived only for the case of one stochastic equation with, usually, time independent coefficients $a, \sigma$.

In $\$ 1$ we prove, under very weak assumptions, that every solution of $(0.1)$ is unbounded with probability 1 . The main results of this paper are given in $\S_{3}$. They are concerned with asymptotic estimates on $\xi(t)$. The most interesting estimates (given in Theorem 5) are of the form

$$
\begin{aligned}
& E|\xi(t)-\bar{\sigma} w(t)|^{2}=o\left(t^{1-\delta}\right) \quad(0 \leq \delta<1), \\
& E|\xi(t)-\bar{\sigma} w(t)|^{2}=O(1) .
\end{aligned}
$$

The estimate $(0.2)$ is proved under the following assumptions: The diffusion matrix $b(t, x)=1 / 2 \sigma(t, x) \sigma *(t, x)$ is positive definite, uniformly for $t \geq 0, x$ in compact sets,

Received by the editors November 8, 1971.

AMS 1969 subject classifications. Primary 6075; Secondary 6030, 6040, 6062, 3516, 3562.

Key words and phrases. Limit behavior, stochastic differential equations, Brownian motion, asymptotic behavior of solutions, diffusion matrix, exit time, parabolic equations, Ito formula, convergence in distribution, Cauchy problem.

(1) This work is partially supported by National Science Foundation grants GP-5558 and GP-28484. 


$$
\begin{aligned}
\left|a_{i}(t, x)\right| & \leq \epsilon(|x|) /(1+|x|)^{1+\delta}, \\
\left|\sigma_{i j}(t, x)-\bar{\sigma}_{i j}\right| & \leq \epsilon(|x|) /(1+|x|)^{\delta}
\end{aligned}
$$

where $\epsilon(r) \rightarrow 0$ if $r \rightarrow \infty$ and $\bar{\sigma}_{i j}$ are constants, and the matrix $\bar{b}=1 / 2 \bar{\sigma} \bar{\sigma}^{*}$ has at least two positive eigenvalues. If $(0.4),(0.5)$ hold with $\delta>1$ and if $\bar{b}$ has at least three positive eigenvalues then $(0.3)$ holds.

Some auxiliary estimates needed in $\$ 3$ are proved in $\$ 2$.

In $\$ 4$ we give some applications of the estimates $(0.2),(0.3)$.

In $\$ 5$ we consider the case $m=1$. It is shown that if $\sigma \equiv 1$ and $a=a(x)$ is in $L^{1}(-\infty, \infty)$, then the estimate $(0.2)$ with $\delta=0$ is true if and only if

$$
\int_{-\infty}^{\infty} a(x) d x=0
$$

The case of a general $\sigma=\sigma(x)$ is similarly treated.

In $\$ 6$ we give an example of a system, with $\sigma=$ identity matrix, for which $a(x)=O((\log |x|) /|x|)$, but for which the estimate

$$
E|\xi(t)|^{2}=O(t)
$$

is false.

In $\$ 7$ we consider equations with unbounded $a, \sigma$ and briefly extend the estimates of $\$ \$ 2,3$.

Acknowledgement. In the original version of the manuscript, the proofs of Theorems 2-5 involved comparison functions constructed by actually solving boundary value problems. Professor S. R. S. Varadhan has pointed out that much simpler comparison functions can be used and that this leads to both much shorter proofs as well as far less restrictive assumptions on the coefficients. The present version of Theorems $2-5$ and their proofs $(\$ \$ 2,3)$ are based on the suggestions of Professor Varadhan.

1. Unboundedness of the solution. We shall deal with the behavior, as $t \rightarrow \infty$, of solutions $\xi(t)$ of stochastic differential systems of $m$ equations

$$
d \xi(t)=a(t, \xi(t)) d t+\sigma(t, \xi(t)) d w(t)
$$

with

$$
\xi(0)=\xi_{0}
$$

here $a=\left(a_{1}, \cdots, a_{m}\right), \sigma=\left(\sigma_{i j}\right)$ is an $m \times m$ matrix and $w(t)=\left(w_{1}(t), \cdots, w_{m}(t)\right)$ where the $w_{i}(t)$ are independent Brownian motions. The sample space will be denoted by $(\Omega, \mathcal{F}, P)$, and a point in $\Omega$ will be denote by $\omega$.

We introduce the diffusion matrix

$$
b(t, x)=1 / 2 \sigma(t, x) \sigma^{*}(t, x) \quad\left(\sigma^{*}=\text { transpose of } \sigma\right)
$$

and set $b(t, x)=\left(b_{i j}(t, x)\right)$. 
We shall use the notation

where $C$ is the matrix $\left(c_{i j}\right)$.

$$
|C|=\left\{\sum_{i, j}\left(c_{i j}\right)^{2}\right\}^{1 / 2}
$$

Suppose the following condition holds:

$\left(A_{0}\right) a(t, x), \sigma(t, x)$ are measurable functions and, for any $T>0, R>0$, there are positive constants $H_{T}, H_{T R}$ such that

$$
\begin{aligned}
|a(t, x)|+|\sigma(t, x)| \leq H_{T}(1+|x|) \quad & \text { if } t \geq 0, x \in R^{m}, \\
|a(t, x)-a(t, \bar{x})|+|\sigma(t, x)-\sigma(t, \bar{x})| & \leq H_{T R}|x-\bar{x}| \\
& \text { if } t \geq 0,|x| \leq R,|\bar{x}| \leq R .
\end{aligned}
$$

Then (see [5], [6]) for any $\xi_{0}$ with $E\left|\xi_{0}\right|^{2}<\infty$ which is independent of $w(t)$, $t \geq 0$, there exists a unique solution of (1.1), (1.2). Another existence theorem [not requiring the Lipschitz continuity of $a, \sigma$ but assuming $a, \sigma$ to be bounded, $\sigma$ continuous and $\sigma \sigma^{*}$ positive definite] is given in [12].

Throughout this paper it is always assumed that $a(t, x), \sigma(t, x)$ are measurable and $\xi(t)$ is a solution of (1.1), (1.2) with $E|\xi(t)|^{2}<\infty$ for all $t \geq 0$. Except for this section, we shall not make the assumption $\left(A_{0}\right)$.

We shall need the following conditions:

$\left(\mathrm{A}_{1}\right) a(t, x)$ and $\sigma(t, x)$ are Hölder continuous in $(t, x)$, uniformly in compact subsets of $[0, \infty) \times R^{m}$.

$\left(\mathrm{A}_{2}\right)$ For any $R>0$ there are positive constants $\gamma_{R}, \Gamma_{R}$ such that, for some $i, 1 \leq i \leq m$,

$$
b_{i i}(t, x) \Gamma_{R}+a_{i}(t, x) \geq \gamma_{R} \quad \text { for all } t \geq 0,|x| \leq R \text { : }
$$

The last inequality is satisfied, for instance, if $b_{i i}(t, x) \geq \lambda, a_{i}(t, x) \leq C$, where $\lambda, C$ are positive constants.

Theorem 1. Assume that $\left(\mathrm{A}_{0}\right)-\left(\mathrm{A}_{2}\right)$ bold and that $b(t, x)$ is a positive definite martix for any $(t, x) \in[0, \infty) \times R^{m}$. Let $\xi(t)$ be the solution of (1.1), (1.2). Then

$$
\limsup _{t \rightarrow \infty}|\xi(t)|=\infty \quad \text { a.s. }
$$

Note that since $\xi(t)$ is a continuous process, the assertion (1.4) is equivalent to the assertion that

$$
\sup _{t>0}|\xi(t)|=\infty \quad \text { a.s. }
$$

Proof. Denote by $\mu$ the probability measure in $R^{m}$ of the random variable $\xi_{0}$. We first consider the case that $\mu$ has compact support $K$. Denote by $B$ any closed ball in $R^{m}$ containing $K$. Denote by $\partial B$ the boundary of $B$. Denote by $r(B)$ the exit time from $B$ of the solution $\xi(t)$, that is, 
$\underline{r}(B)=$ first $\bar{t}$ such that $\xi(t) \in B$ if $t \leq \bar{t}$, but $\xi\left(t_{n}\right) \notin B$ for a sequence $\left\{t_{n}\right\}$, $t_{n} \downarrow \bar{t}$ if $n \nearrow \infty$

$\gamma(B)=\infty$ if no such $\bar{t}$ exists.

For any $T>0$, let

$$
\tau_{T}(B)=\min \{\tau(B), T\} .
$$

Set $B_{T}=\{t=T\} \times B, S_{T}=\{0<t<T\} \times \partial B$, and let $\phi(t, x)$ be the solution of

$$
\begin{aligned}
L \phi & \equiv \frac{\partial \phi}{\partial t}+\sum_{i, j=1}^{m} b_{i j}(t, x) \frac{\partial^{2} \phi}{\partial x_{i} \partial x_{j}}+\sum_{i=1}^{m} a_{i}(t, x) \frac{\partial \phi}{\partial x_{i}}=-1 \quad \text { in }[0, T) \times B, \\
\phi & =0 \quad \text { on } B_{T} \cup S_{T}
\end{aligned}
$$

the existence and uniqueness of $\phi$ is proved in [4].

We shall prove that

$$
E \tau_{T}(B)=\int_{K} \phi(0, x) d \mu(x) .
$$

By Ito's formula, if $\psi(t, x)$ is a function such that $\psi, D_{x} \psi, D_{x}^{2} \psi$ are continuous for $(t, x) \in[0, T] \times R^{m}\left(D_{x} \psi=\operatorname{grad}_{x} \psi, D_{x}^{2} \psi=\operatorname{grad}_{x}\left(D_{x} \psi\right)\right)$, then, for any $0<t \leq T$,

$$
\begin{aligned}
\psi(t, \xi(t))-\psi\left(0, \xi_{0}\right)= & \int_{0}^{t} D_{x} \psi(s, \xi(s)) \cdot \sigma(s, \xi(s)) d w(s) \\
+ & \int_{0}^{t}\left[\sum_{i=1}^{m} a_{i}(s, \xi(s)) \frac{\partial \psi(s, \xi(s))}{\partial x_{i}}\right. \\
& \left.+\sum_{i, j=1}^{m} b_{i j}(s, \xi(s)) \frac{\partial^{2} \psi(s, \xi(s))}{\partial x_{i} \partial x_{j}}+\frac{\partial \psi(s, \xi(s))}{\partial s}\right] d s .
\end{aligned}
$$

Substituting $t=\tau_{T}(B)$ we get

$$
\begin{aligned}
& \psi\left(\tau_{T}(B), \xi\left(\tau_{T}(B)\right)\right)-\psi\left(0, \xi_{0}\right) \\
& \quad=\int_{0}^{\tau} T^{(B)} D_{x} \psi(s, \xi(s)) \cdot \sigma(s, \xi(s)) d w(s)+\int_{0}^{\tau} T^{(B)}(L \psi)(s, \xi(s)) d s .
\end{aligned}
$$

This formula remains true if $\psi_{t}, D_{x} \psi, D_{x}^{2} \psi$ are only assumed to be continuous in $[0, T] \times B_{0}$ where $B_{0}$ is a ball such that int $B_{0} \supset B$. Indeed, denote by $\widetilde{\psi}$ a function that agrees with $\psi$ in $[0, T] \times B$ and for which $\tilde{\psi}_{t}, D_{x} \tilde{\psi}, D_{x}^{2} \widetilde{\psi}$ are continuous in $[0, T] \times R^{m}$. Since (1.10) holds with $\psi$ replaced by $\tilde{\psi}$, and since each term in $(1.10)$ is equal to the corresponding term with $\psi$ replaced by $\tilde{\psi},(1.10)$ follows for $\psi$.

We shall now use (1.10) with $\psi=\psi_{\epsilon}$ where $\psi_{\epsilon}$ is the solution of (1.7) with 
$B$ replaced by the ball $B_{\epsilon}$ which is concentric with $B$ and has radius $r_{0}+\epsilon, r_{0}=$ radius of $B$. Taking the expectation on both sides of (1.10), we then get

$$
E \psi_{\epsilon}\left(\tau_{T}(B), \xi\left(\tau_{T}(B)\right)\right)-E \psi_{\epsilon}\left(0, \xi_{0}\right)=E \int_{0}^{\tau} T^{(B)}(-1) d s=-E \tau_{T}(B)
$$

note that the expectation of the stochastic integral vanishes.

By standard arguments from [4] one can show that, as $\epsilon \rightarrow 0, \psi \epsilon(t, x) \rightarrow \phi(t, x)$ uniformly in $[0, T] \times B$. Hence, $(1.11)$ yields

$$
E \phi\left(\tau_{T}(B), \xi\left(\tau_{T}(B)\right)\right)-E \phi\left(0, \xi_{0}\right)=-E \tau_{T}(B) .
$$

Since $\left(\tau_{T}(B), \xi\left(\tau_{T}(B)\right)\right)$ belongs to $B_{T} \cup S_{T}$ for all $\omega \in \Omega, \phi\left(\tau_{T}(B), \xi\left(\tau_{T}(B)\right)\right)=0$. We thus get $E \tau_{T}(B)=E \phi\left(0, \xi_{0}\right)$; this is the assertion (1.8).

Now let $\lambda(x)$ be a function satisfying, for all $t \geq 0, L_{\chi} \leq-1$ in $[0, T] \times B$, $0 \leq \chi(x) \leq C$ in $B$, where $C$ is a positive constant. If $1+x_{i} \leq x_{i}^{0}$ for all $\left(x_{1}, x_{2}, \cdots, x_{m}\right) \in B\left(i\right.$ as in $\left.\left(A_{2}\right)\right)$, then we can take

$$
\chi=A\left(\exp \left(a x_{i}^{0}\right)-\exp \left(\alpha x_{i}\right)\right)
$$

where $\alpha, A$ are suitable positve constants; here we use the condition $\left(A_{2}\right)$.

We have $L(\phi-\chi) \geq 0$ in $[0, T) \times B, \phi-\chi \leq 0$ on $B_{T} \cup S_{T}$. Hence, by the maximum principle, $\phi-\chi \leq 0$ in $[0, T] \times B$. Consequently,

$$
0 \leq \phi(t, x) \leq C ; \quad C \text { independent of } T .
$$

From (1.8), (1.12) we deduce that $E \tau_{T}(B) \leq C$.

Now, $\tau_{T}(B) \nearrow$ if $T \nearrow$. Using the monotone convergence theorem, we conclude that $\tau(B)=\lim _{T \rightarrow \infty} \tau_{T}(B)$ satisfies $E \tau(B) \leq C$. In particular,

$$
\tau(B)<\infty \quad \text { a.s. }
$$

Now take an increasing sequence of closed balls $B_{n}$ with center 0 and radii $R_{n} \rightarrow \infty$. Let

$$
M_{n}=\left\{\sup _{t>0}|\xi(t)|>R_{n}\right\}, \quad M=\bigcap_{n=1}^{\infty} M_{n} .
$$

Denote by $\chi_{n}$ the indicator function of $B_{n}$, and let $\xi_{n}(t)$ be the solution of (1.1) with the initial condition $\xi_{n}(0)=\chi_{n} \xi_{0}$; here the condition $\left(A_{0}\right)$ is first used. Denote by $\tau_{n}\left(B_{n}\right)$ the exit time from $B_{n}$ of the solution $\xi_{n}(t)$. As is well known (see [6]), $\tau_{n}\left(B_{n}\right)=\tau\left(B_{n}\right)$ a.s. and $\xi_{n}(t)=\xi(t)$ if $0 \leq t \leq \tau\left(B_{n}\right)$. Hence,

$$
P\left\{\sup _{t>0}|\xi(t)|>R_{n}\right\}=P\left\{\sup _{t>0}\left|\xi_{n}(t)\right|>R_{n}\right\},
$$

and the right-hand side is equal to 1 , by (1.13) with $K=\dot{B}=B_{n}$. We conclude that $P\left(M_{n}\right)=1$. Since the sequence $\left\{M_{n}\right\}$ is monotone decreasing to $M$,

$$
P(M)=\lim _{n \rightarrow \infty} P\left(M_{n}\right)=1
$$


Now, if $\omega \in M$, then $\omega \in M_{n}$ for all $n$, so that

$$
\sup _{t>0}|\xi(t)|>R_{n} \text { for all } n,
$$

i.e. (1.5) holds. This completes the proof.

2. Auxiliary results.

Theorem 2. Assume that

$$
\sum_{i=1}^{m} b_{i i}(t, x) \leq C, \quad \sum_{i=1}^{m} x_{i} a_{i}(t, x) \leq C,
$$

where $C$ is a positive constant. Then

$$
E|\xi(t)|^{2} \leq K t+K^{\prime} \quad \text { for all } t \geq 0,
$$

where $K, K^{\prime}$ are positive constants.

Proof. Using Ito's formula with $\phi(x)=|x|^{2}, x=\xi(t)$ and taking the expectation, we get

$$
E|\xi(t)|^{2}=E\left|\xi_{0}\right|^{2}+E \int_{0}^{t} g(s, \xi(s)) d s
$$

where

$$
g(t, x)=L \phi=2 \sum b_{i i}(t, x)+2 \sum x_{i} a_{i}(t, x) .
$$

Since, by our assumptions, $g^{\prime}(t, x) \leq C_{1}$ where $C_{1}$ is a positive constant, (2.1) follows.

Notation. We shall denote the eigenvalues of $b(t, x)$ by $\lambda_{i}(t, x)$, where

$$
\lambda_{1}(t, x) \leq \lambda_{2}(t, x) \leq \cdots \leq \lambda_{m}(t, x) .
$$

Lemma 1. Assume that

$$
\sum_{i, j}\left|b_{i j}(t, x)\right| \leq C \quad \text { for } t \geq 0, x \in R^{m} \text { (C constant); }
$$

for any $R>0$ there is $\mu(R)>0$ such that

$$
\sum_{i, j} b_{i j}(t, x) \xi_{i} \xi_{j} \geq \mu(R)|\xi|^{2} \quad \text { if } t \geq 0,|x| \leq R, \xi \in R^{m}
$$

$(2.4)^{(1+|x|)} \sum_{i}\left|a_{i}(t, x)\right| \leq \epsilon(|x|)$ for $t \geq 0, x \in R^{m}$, where $\epsilon(r) \rightarrow 0$ if $r \rightarrow \infty$;

$$
\left.\lambda_{m}(t, x) \geq \gamma>0 \text { for } t \geq 0, x \in R^{m} \text { ( } \gamma \text { constant }\right) .
$$

Let $\phi(t, x)$ be a bounded measurable function sucb that $\phi(t, x)=0$ if $x \notin \Omega$ where $\Omega$ is a compact set. Then, for any $\eta>0$,

$$
E\left|\int_{0}^{t} \phi(s, \xi(s)) d s\right| \leq K_{1}+K_{2} t^{(1+\eta) \cdot / 2}
$$

where $K_{1}, K_{2}$ are positive constants. If furtber, 


$$
\lambda_{m-1}(t, x) \geq \gamma^{\prime}>0 \quad \text { for } t \geq 0, x \in R^{m}\left(\gamma^{\prime} \text { constant }\right)
$$

Then (2.6) bolds for some $-1<\eta<0$.

Proof. Since a translation in the $x$-space does not change the assumptions and assertions of the lemma, we may assume that $x=0$ is not in $\Omega$. But then there is a continuous function $\psi(r)$ such that

$$
|\phi(t, x)| \leq \psi(|x|)
$$

and $\psi(r)=0$ if $r \leq r_{0}$ or if $r \geq r_{1}$ where $0<r_{0}<r_{1}<\infty$. We shall construct a function $F(r)$ such that $f(x)=F(|x|)$ is in $C^{2}\left(R^{m}\right)$ and

$$
(L f)(t, x) \geq \psi(|x|) .
$$

Note first that

$$
L f(r)=A(t, x) F^{\prime \prime}(r)+\left(F^{\prime}(r) / r\right)[B(t, x)-A(t, x)+C(t, x)]
$$

where

$$
\begin{aligned}
& A(t, x)=\frac{1}{r^{2}} \sum_{i, j} b_{i j}(t, x) x_{i} x_{j}, \\
& B(t, x)=\sum_{i} b_{i i}(t, x), \\
& C(t, x)=\sum_{i} x_{i} a_{i}(t, x) .
\end{aligned}
$$

Let $\theta(r)$ be a continuous function, vanishing for $r<r_{0} / 2$ and satisfying, for $r \geq r_{0}$

$$
(1+\theta(r)) A(t, x) \leq B(t, x)+C(t, x)
$$

Since, for any $R>0, A(t, x) \geq \mu(R)>0$ if $t \geq 0,|x| \leq R$, and since $B(t, x) \geq 0$, $|C(t, x)|$ is bounded, one can certainly construct such a function $\theta(r)$. Noting that

$$
\lambda_{1}(t, x) \leq A(t, x) \leq \lambda_{m}(t, x), \quad B(t, x)=\lambda_{1}(t, x)+\cdots+\lambda_{m}(t, x)
$$

and using (2.5) and (2.4), we can construct $\theta(r)$ such that

$$
\theta(r)=-\eta \quad \text { if } r \text { is sufficiently large (for any } \eta>0 \text { ). }
$$

If (2.7) holds then we can take

$$
\theta(r)=-\eta \text { if } r \text { is sufficiently large (for some }-1<\eta<0 \text { ); }
$$

in fact, we can take any $\eta$ such that

$$
-\eta<\gamma^{\prime} / \delta^{\prime}, \quad \delta^{\prime}=\limsup _{|x| \rightarrow \infty}\left\{\sup _{t \geq 0} \lambda_{m}(t, x)\right\} .
$$


Introduce the functions $I(s)=\int_{r_{0}}^{s}(\theta(u) / u) d u$,

$$
f(x)=F(r)=\frac{1}{\lambda} \int_{0}^{r} e^{-I(s)} d s \int_{0}^{s} e^{I(\tau)} \psi(\tau) d \tau
$$

for some $\lambda>0, r=|x|$. Since $F$ is in $C^{2}$ and $F(r)=0$ if $r \leq r_{0}, f(x)$ is in $C^{2}\left(R^{m}\right)$. Notice that

$$
F^{\prime \prime}(r)+(\theta(r) / r) F^{\prime}(r)=(1 / \lambda) \psi(r)
$$

and that $F^{\prime}(r) \geq 0$. Using (2.10), (2.11) we then get

$$
L f(x) \geq\left[F^{\prime \prime}(r)+(\theta(r) / r) F^{\prime}(r)\right] A(t, x) \geq(A(t, x) / \lambda) \psi(r) \geq \psi(r)
$$

if $\lambda=\mu\left(r_{1}\right)$ (so that $A(t, x) \geq \lambda$ if $t \geq 0,|x| \leq r_{1}$ ).

Having proved (2.9), we now use (2.8) and Ito's formula to get

$$
\begin{aligned}
& E\left|\int_{0}^{t} \phi(s, \xi(s)) d s\right| \leq E \int_{0}^{t} \psi(\xi(s)) d s \\
& \quad \leq E \int_{0}^{t}(L f)(s, \xi(s)) d s=E F(|\xi(t)|)-E F\left(\left|\xi_{0}\right|\right) \leq E F(|\xi(t)|) .
\end{aligned}
$$

Noting that $F(r) \leq C r^{1+\eta}$, we obtain

$$
E\left|\int_{0}^{t} \phi(s, \xi(s)) d s\right| \leq C E|\xi(t)|^{1+\eta}+C \leq C\left\{E|\xi(t)|^{2}\right\}^{(1+\eta) / 2}+C
$$

for suitable constants $C$. Using Theorem 2, the assertion (2.6) follows.

Remark 1. If (2.2), (2.3), (2.5) hold and if (2.4) is replaced by

$$
(1+|x|)^{1+\delta} \sum_{i}\left|a_{i}(t, x)\right| \leq \epsilon(|x|), \quad \delta>0, \epsilon(r) \rightarrow+\text { if } r \rightarrow 0,
$$

then we can construct $\theta(r)$ such that, for large $\left.r, \theta^{\prime} r\right)=-D / r^{\beta}$ for any $0<\beta \leq \delta$ and a positive constant $D$. It follows that $F(r) \leq C r$, so that $(2.6)$ is valid with $\eta=0$.

Remark 2. If the conditions (2.2), (2.3), (2.16) hold and if (2.5) is replaced by

$$
\lambda_{m}(t, x) \geq c /(1+|x|)^{\delta}, \quad c>0, \delta>0,
$$

then we can again take $\theta_{r}$ ) such that (2.12) holds. Consequently, (2.6) is valid for any $\eta>0$. Note that the condition (2.17) is equivalent to the condition

$$
\sum_{i} b_{i i}(t, x) \geq e /(1+|x|)^{\delta} \text { for some } e>0
$$

We shall next extend Lemma 1 to the case where $\phi(t, x)$ does not necessarily vanish if $|x|$ is large, but either

$$
|\phi(t, x)-\Phi| \leq C /(1+|x|)^{\alpha} \quad(\alpha>0)
$$

or

$$
|\phi(t, x)-\Phi| \leq \epsilon(|x|) /(1+|x|)^{\alpha} \quad(\alpha \geq 0), \epsilon(r) \rightarrow 0 \text { if } r \rightarrow 0 ;
$$

here $\Phi$ is a constant. 
Lemma 2. Let the conditions (2.2)-(2.5) bold and let $\phi(t, x)$ be a bounded measurable function satisfying (2.18). Then

$$
\left|E \int_{0}^{t} \phi(s, \xi(s)) d s-\Phi t\right|=O\left(t^{(1+\eta) / 2}\right)+O\left(t^{1-\alpha / 2}\right)
$$

for any $\eta>0$; if (2.7) also bolds then (2.20) bolds for some $-1<\eta<0$. If the function $\phi(t, x)$ satisfies (2.19) instead of (2.18), then

$$
E\left|\int_{0}^{t} \phi(s, \xi(s)) d s-\Phi t\right|=O\left(t^{(1+\eta) / 2}\right)+o\left(t^{1-\alpha / 2}\right)
$$

with the same $\eta$ as before.

Proof. Suppose first that (2.19) holds. Then, if any $\epsilon>0$, there exists an $R_{\epsilon}>0$ such that

$$
|\phi(t, x)-\Phi|<\epsilon / r^{\alpha} \text { if }|x|>R_{\epsilon}, t \geq 0 .
$$

Let $\chi_{\epsilon}(x)=1$ if $|x|<R_{\epsilon}, \chi_{\epsilon}(x)=0$ if $|x| \geq R_{\epsilon}$, and write

By Lemma 1,

$$
\begin{aligned}
\phi(t, x)-\Phi & =[\phi(t, x)-\Phi] \chi_{\epsilon}(x)+[\phi(t, x)-\Phi]\left[1-\chi_{\epsilon}(x)\right] \\
& \equiv \phi_{1}(t, x)+\phi_{2}(t, x) .
\end{aligned}
$$

$$
E\left|\int_{0}^{t} \phi_{1}(s, \xi(s)) d s\right|=O\left(t^{(1+\eta) / 2}\right) .
$$

Next, let $\psi(r)$ be a continuous function such that

$$
\begin{aligned}
& \psi(r)=0 \quad \text { if } 0<r<r_{0} / 2, \\
& \psi(r)=\epsilon / r^{a} \text { if } r>r_{0},
\end{aligned}
$$

for some $0<r_{0}<R_{\epsilon}$. We shall slightly modify the definition of $\theta(r)$ by replacing the condition $(2.11)$ by the stricter condition

$$
(1+\theta(r)) A(t, x) \leq \rho B(t, x)+C^{\prime}(t, x)
$$

where $\rho$ is any positive number $<1$. We still have (2.12) for any $\eta=\eta(\rho)>0$ provided $\rho$ is sufficiently close to 1 ; if (2.7) holds then we can make (2.13) hold if $\rho$ is sufficiently close to 1 .

Let $f(x)$ be defined by (2.14), with $\lambda>0$ to be determined later. Then, by (2.10), (2.25),

$$
\begin{aligned}
L f(x) & \geq\left[F^{\prime \prime}(r)+\frac{\theta(r)}{r} F^{\prime}(r)\right] A(t, x)+\frac{F^{\prime}(r)}{r}(1-\rho) B(t, x) \\
& \geq \frac{F^{\prime}(r)}{r}(1-\rho) B(t, x) \geq \frac{c}{\lambda} \frac{1}{r} e^{-I(r)} \int_{r_{0}}^{r} e^{I(s)} \frac{\epsilon}{s^{\alpha}} d s \\
& \geq \frac{c}{\lambda} \frac{\epsilon}{r^{1+\alpha}} e^{-I(r)} \int_{r_{0}}^{r} e^{I(s)} d s
\end{aligned}
$$


where $c$ is any positive constant $\leq(1-\rho) B(t, x)$. Since $e^{I(r)} \sim r^{-\eta}$ if $r \rightarrow \infty$, we get

$$
L f(x) \geq-\frac{c_{1}}{\lambda} \frac{\epsilon}{r^{\alpha}} \geq\left|\phi_{2}(t, x)\right| \quad\left(|x| \geq R_{\epsilon}\right)
$$

where $c_{1}$ is a positive constant (independent of $\epsilon$ ) provided we take $\lambda \leq c_{1}$. If $|x|<R_{\epsilon}$ then clearly $L f(x) \geq 0=\left|\phi_{2}(t, x)\right|$.

By (2.27) and Ito's formula,

$$
\begin{aligned}
& E\left|\int_{0}^{t} \phi_{2}(s, \xi(s)) d s\right| \leq E \int_{0}^{t}\left|\phi_{2}(s, \xi(s))\right| d s \\
& \leq E \int_{0}^{t}(L f)(s, \xi(s)) d s=E F(|\xi(t)|)-E F\left(\left|\xi_{0}\right|\right) \leq E F(|\xi(t)|) .
\end{aligned}
$$

Since

$$
F(r) \leq \begin{cases}C_{\epsilon} r^{2-\alpha}+C & \text { if } 2-a>1+\eta, \\ C \epsilon r^{1+\eta}+C & \text { if } 2-a<1+\eta,\end{cases}
$$

and since we may assume that that $\eta$ is such that $a+\eta \neq 1$, we deduce, after using Hölder's inequality and Theorem 2, that

$$
E F(|\xi(t)|) \leq \begin{cases}C \epsilon t^{1-\alpha / 2}+C & \text { if } \alpha+\eta<1 \\ C \epsilon t^{(1+\eta) / 2}+C & \text { if } \alpha+\eta>1\end{cases}
$$

Substituting this into (2.28) and combining with (2.23), (2.22), we get

$$
E\left|\int_{0}^{t} \phi(s, \xi(s)) d s-\Phi t\right| \leq C_{0} t^{(1+\eta) / 2}+C_{1} \epsilon t^{1-\alpha / 2}
$$

$C_{1}$ is a constant independent of $\epsilon$. Since $\epsilon$ is arbitrary, (2.29) implies the assertion (2.21) with $\eta$ replaced by $\eta^{\prime}$, for any $\eta^{\prime}>\eta$. But this clearly completes the proof of (2.21). The proof of (2.20) (for $\phi$ satisfying (2.18)) is similar.

Remark 1. Let the condition (2.4) be replaced by the stricter condition (2.16). Then we can modify the proof of Lemma 2 with $\epsilon=C$, in case (2.5) holds, taking, for any $0<\beta \leq \delta$,

$$
1-\rho=(D / 2) / r^{\beta}, \quad \theta(r)=D / r^{\beta},
$$

for large $r$, where $D$ is a positive constant; in (2.14) we replace $\psi(\tau)$ by $\psi(\tau) \tau_{\tau} \beta$, so that in (2.26) $\alpha$ is replaced by $\alpha-\beta$. We conclude that if $(2.18)$ holds then

$$
E\left|\int_{0}^{t} \phi(s, \xi(s)) d s-\Phi t\right|=O\left(t^{1 / 2}\right)+O\left(t^{1-(\alpha-\beta) / 2}\right)
$$

for any $\beta>0$.

Remark 2. Suppose now that (2.5) is replaced by (2.17) and that (2.2), (2.3) and (2.16) hold. Modifying the proof of Lemma 2 (by replacing $\psi(\tau)$ by $\psi(\tau) \tau^{\delta}$ in (2.14) and $\alpha$ by $\alpha-\delta$ in (2.26)) we conclude that if (2.19) holds then 


$$
E\left|\int_{0}^{t} \dot{\phi}(s, \xi(s)) d s-\Phi t\right|=O\left(t^{(1+\eta) / 2}\right)+o\left(t^{1-(\alpha-\delta) / 2}\right)
$$

for any $\eta>0$. If $\phi$ satisfies (2.18) then (2.31) holds with " 0 " replaced by " $O$ ".

Our next concern is to evaluate more precisely the $\eta$ occurring in Lemma 2. We shall assume that, for all $i, j$,

$$
\lim _{|x| \rightarrow \infty} h_{i j}(t, x)=\bar{b}_{i j} \quad \text { uniformly with respect to } t,
$$

where $\bar{b}_{i j}$ are constant. We then introduce the notation:

$$
d=\text { number of positive eigenvalues of } \bar{b}=\left(\bar{b}_{i j}\right) \text {. }
$$

Observe that if (2.3) holds then (2.5) is equivalent to $d \geq 1$, and (2.7) is equivalent to $d \geq 2$.

We now note that the assumptions and assertions of Lemma 2 remain unchanged if a nonsingular affine transformation $x \rightarrow A x$ is performed. But such a transformation changes $b(t, x)$ into $A b(t, x) A^{*}$. We can, therefore, choose $A$ such that

$$
\begin{aligned}
& \bar{b}_{i j}=0 \quad \text { if } \quad i \neq j, \\
& \bar{b}_{i i}=1 \quad \text { if } \quad i=1,2, \ldots, d, \\
& \bar{b}_{i i}=0 \quad \text { if } i=d+1, \ldots, m ;
\end{aligned}
$$

$d=0$ means that $\bar{b}_{i i}=0$ for all $i$, and $d=m$ means that $\bar{b}_{i i}=1$ for all $i$.

If $d \geq 2$ then we can take, in the proofs of Lemmas $1,2, \theta(r)=\nu$ for any $\nu<1$ and all large $r$. This leads to the assertions of Lemmas 1,2 with any $-1<\eta<0$.

If $d \geq 3$ then we can take $\theta(r)=3 / 2$ for all large $r$. This leads to the assertion of Lemma 1 with $\eta=-1$. If $\phi$ satisfies (2.18) then, instead of (2.20), we have

$$
E\left|\int_{0}^{t} \phi(s, \xi(s)) d s-\Phi t\right| \leq \begin{cases}O(1)+O\left(t^{1-\alpha / 2}\right) & \text { if } a \neq 2 \\ O(\log t) & \text { if } \alpha=2\end{cases}
$$

If $d \geq 2$ and if (2.16) holds and

$$
\sum_{i, j}\left|b_{i j}(t, x)-\bar{b}_{i j}\right| \leq \epsilon(|x|) /(1+|x|)^{\delta}, \quad \delta>0, \epsilon(r) \rightarrow 0 \text { if } r \rightarrow \infty,
$$

then we can take, in the proof of Lemma $1, \theta(r)=1-D / r^{\beta}$ (for any $0<\beta \leq \delta$ ) for $r$ large, where $D$ is a positive constant. This leads to the assertion of Lemma 1 with $O\left(t^{(1+\eta) / 2}\right)$ replaced by $O(\log t)$. We can use the same $\theta(r)$ also in the proof of Lemma 2 (cf. Remark 1 following Lemma 2). It follows that if $\phi$ satisfies (2.18) then

$$
E\left|\int_{0}^{t} \phi(s, \xi(s)) d s-\Phi t\right|=O(\log t)+O\left(t^{1-(\alpha-\beta) / 2}\right)
$$


for any $\beta>0$.

If $d \geq 1$ and (2.16), (2.35) hold, then we can take, in the proof of Lemma 1 , $\theta(r)=-D / r^{\beta}$ (for any $0<\beta \leq \delta$ ) for large $r$ where $D$ is a positive constant. We conclude that Lemma 1 holds with $\eta=0$. As for Lemma 2, if (2.18) holds, then

$$
E\left|\int_{0}^{t} \phi(s, \xi(s)) d s-\Phi t\right|=O\left(t^{1 / 2}\right)+O\left(t^{1-(a-\beta) / 2}\right)
$$

for any $\beta>0$.

We sum up:

Lemma 3. (a) Let the conditions (2.2)-(2.4), (2.32) bold and let $d \geq 2$. Let $\phi(t, x)$ be a bounded measurable function. If $\phi$ satisfies (2.18) then (2.20) bolds for any $-1<\eta<0$, and if $\phi$ satisfies (2.19) then (2.21) bolds with any $-1<\eta<0$.

(b) Let the conditions (2.2)-(2.4), (2.32) bold and let $d \geq 3$. Let $\phi(t, x)$ be a bounded measurable function satisfying (2.18). Then (2.34) bolds.

(c) Let the conditions (2.2)-(2.4), (2.35) and (2.16) bold and let $d \geq 2$. Let $\phi(t, x)$ be a bounded measurable function sat isfying (2.18). Then (2.36) bolds.

(d) Let the conditions (2.2)-(2.4), (2.16), (2.35) bold and let $d \geq 1$. Let $\phi(t, x)$ be a bounded measurable function satisfying (2.18). Then (2.37) bolds.

3. Asymptotic estimates.

Theorem 3. Let the conditions (2.2)-(2.5) bold. Then

$$
E|\xi(t)|^{2} \geq K t-K^{\prime} \quad \text { for all } t \geq 0
$$

where $K, K^{\prime}$ are positive constants.

Proof.

$$
L|x|^{2}=2 \sum b_{i i}(t, x)+2 \sum x_{i} a_{i}(t, x) \geq \gamma-\phi(x)
$$

where $\phi(x)$ is a bounded, measurable and nonnegative function having compact support. Hence,

$$
E|\xi(t)|^{2}-E\left|\xi_{0}\right|^{2}=E \int_{0}^{t}\left(L|x|^{2}\right)(s, \xi(s)) d s \geq \gamma t-E \int_{0}^{t} \phi(\xi(s)) d s
$$

and, by Lemma 1 ,

$$
E \int_{0}^{t} \phi(\xi(s)) d s=o(t)
$$

This yields the inequality (3.1).

Remark. Lemma 1 and Theorem 1 remain true if the condition (2.4) is replaced by the weaker condition

$$
(1+|x|) \sum_{i}\left|a_{i}(t, x)\right| \leq \epsilon_{0}, .
$$

$\epsilon_{0}$ a sufficiently small, positive constant. 
Theorem 4. Let (2.2), (2.3), (2.16), (2.32) bold and let $d \geq 1$. Then

$$
E|\xi(t)|^{2}=2(\operatorname{tr} \bar{b}) t+O\left(t^{(1+\eta) / 2}\right)+o\left(t^{1-\delta / 2}\right)
$$

where $\operatorname{tr} \bar{b}=\Sigma_{i} \bar{b}_{i i}$ and $\eta$ is any positive number; if $d \geq 2$ then $\eta$ is any number $>-1$.

When $\delta=0$ we get

Corollary. Let (2.2)-(2.4), (2.32) bold and let $d \geq 1$ : Then

$$
E|\xi(t)|^{2}=2(\operatorname{tr} \bar{b}) t+o(t)
$$

Proof of Theorem 4. Clearly,

$$
\left.|L| x\right|^{2}-2 \operatorname{tr} \bar{b} \mid \leq \epsilon^{\prime}(|x|) /(1+|x|)^{\delta}
$$

where $\epsilon^{\prime \prime}(r) \rightarrow 0$ if $r \rightarrow \infty$. Hence, by Lemmas 2 and $3(a)$ with $\phi(t, x)=|x|^{2}$, $\Phi=2 \operatorname{tr} \bar{b}$,

$$
\begin{aligned}
E|\xi(t)|^{2}-E\left|\xi_{0}\right|^{2} & =E \int_{0}^{t}\left(L|x|^{2}\right)(s, \xi(s)) d s \\
& =2(\operatorname{tr} \bar{b}) t+O\left(t^{(1+\eta) / 2}\right)+O\left(t^{1-\delta / 2}\right)
\end{aligned}
$$

where $\eta$ is any positive number if $d \geq 1$ and any number $>-1$ if $d \geq 2$. This yields (3.3).

Remark 1. Using Lemma 3 one can give variants of (3.3) in case (2.16) holds with $\epsilon(r)$ replaced by a positive constant.

Remark 2. By a slight change in the proof of Theorem 4 one derives the estimate

$$
E|\langle e, \xi(t)\rangle|^{2}=2\langle e, \bar{b} e\rangle+O\left(t^{(1+\eta) / 2}\right)+o\left(t^{1-\delta / 2}\right)
$$

where $e$ is any unit vector.

Lemma 4: Let the conditions (2.2), (2.3), (2.32) and (2.16) with $0 \leq \delta<1$ bold, and let $d \geq 2$. Then

$$
E\left|\int_{0}^{t} a_{j}(s, \xi(s)) d s\right|^{2}=o\left(t^{1-\delta}\right)
$$

Proof. For any $\epsilon>0$ we can write

$$
\left|a_{j}(t, x)\right| \leq g_{1}(x)+g_{2}(|x|)
$$

where the $g_{i}$ are bounded measurable functions; $g_{1}(x)$ has a compact support,

$$
g_{2}(r)=\epsilon /(1+r)^{1+\delta} \quad \text { if } r>r_{1}
$$

and $g_{2}(r)=0$ if $r \leq r_{1}$ for some $0<r_{1}<\infty$. We have

$$
E\left|\int_{0}^{t} a_{j}(s, \xi(s)) d s\right|^{2} \leq 2 E\left|\int_{0}^{t} g_{1}(\xi(s)) d s\right|^{2}+2 E\left|\int_{0}^{t} g_{2}(|\xi(s)|) d s\right|^{2}
$$


Let $F(r)$ be the function constructed in the proof of Lemma 1 for $\phi=g_{1}$. Then, for large $r$,

$$
F(r) \leq c r^{1+\eta}, \quad F^{\prime}(r) \leq C r^{\eta}
$$

for any $-1<\eta<0$. Hence, by Ito's formula,

$$
\left|\int_{0}^{t} g_{1}(\xi(s)) d s\right| \leq \int_{0}^{t}(L F)(s, \xi(s)) d s
$$

so that

$$
\leq F\left(|\xi(t)|^{1+\eta}\right)-\int_{0}^{t} \nabla_{x} F \cdot \sigma(s, \xi(s)) d w(s),
$$

$$
E\left|\int_{0}^{t} g_{1}(\xi(s)) d s\right|^{2} \leq 2 E F(|\xi(t)|)^{2}+2 E \int_{0}^{t}\left|\nabla_{x} F \cdot \sigma\right|^{2} d s
$$

Now, for large $r$,

$$
\leq C t^{1+\eta}+C+2 E \int_{0}^{t}\left|\nabla_{x} F \cdot \sigma\right|^{2} d s
$$

$$
\left|\nabla_{x} F \cdot \sigma\right|^{2} \leq C r^{2 \eta} \quad(-1<\eta<0)
$$

Hence, by Lemma 3(a) with $a=-2 \eta$,

and (3.10) gives

$$
E \int_{0}^{t}\left|\nabla_{x} F \cdot \sigma\right|^{2} d s \leq C t^{1+\eta}+C
$$

$$
E\left|\int_{0}^{t} g_{1}(\xi(s)) d s\right|^{2} \leq C t^{1+\eta}+C
$$

Next let $F_{0}(r)$ be the function $F^{\prime}(r)$ constructed in Lemma 2 for $\phi_{2}=g_{2}$. We can take $-1<\eta<-\delta$. Then

$$
F_{0}(r) \leq C \epsilon r^{1-\delta}+C \epsilon, \quad F_{0}^{\prime}(r) \leq C \epsilon r^{-\delta}+C \epsilon,
$$

where $C$ is a constant independent of $\epsilon$. Analogously to (3.10), (3.11) we get

$$
E\left|\int_{0}^{t} g_{2}(|\xi(s)|) d s\right|^{2} \leq C \epsilon t^{1-\delta}+C+2 E \int_{0}^{t}\left|\nabla_{x} F_{0} \cdot \sigma\right|^{2} d s,
$$

$$
\left|\nabla_{x} F_{0} \cdot \sigma\right|^{2} \leq C \epsilon^{2} / r^{2 \delta}
$$

By Lemma 3(a),

$$
E \int_{0}^{t}\left|\nabla_{x} F_{0} \cdot \sigma\right|^{2} d s \leq C t^{(1+\eta) / 2}+C \epsilon^{2} t^{1-\delta}+C
$$

Hence,

$$
E\left|\int_{0}^{t} g_{2}(|\xi(s)|) d s\right|^{2} \leq C \epsilon t^{1-\delta}+C t^{(1+\eta) / 2}+C .
$$

Combining this with (3.13), (3.8), we get

$$
E\left|\int_{0}^{t} a_{j}(s, \xi(s)) d s\right|^{2} \leq C t^{1+\eta}+C t^{1-\delta}+C .
$$


This gives the assertion of the lemma.

We shall now modify the proof of Lemma 4 in the case where $d \geq 3$ and (2.16) holds with $\delta>1$.

The function $F(r)$ occurring in (3.9) is given by

$$
F(r)=\int_{\rho_{0}}^{r} e^{-I(s)} d s \int_{\rho_{0}}^{s} e^{I(\tau)} \bar{\gamma}(\tau) d \tau \text { if } r>\rho_{0},
$$

where $\bar{\gamma}(\tau)=0$ if $\tau>\rho_{1}$, for some $0<\rho_{0}<\rho_{1}<\infty$; note that $r$ is not to be taken as $|x|$ but rather as $\left|x-x^{0}\right|$ for some $x^{0}$ outside the support of $g_{1}(x)$. We can take $\eta(r)=3 / 2$ for $r$ large, so that $I(r) \sim \log r^{3 / 2}$. Hence

$$
F(r) \leq C, \quad F^{\prime}(r) \leq C / r^{3 / 2}
$$

instead of (3.9). Hence (3.11) is replaced by

$$
\left|\nabla_{x} F \cdot \sigma\right| \leq C / r^{3} \text {. }
$$

Lemma 3(b) with $a=3$ gives

$$
E \int_{0}^{t}\left|\nabla_{x} F \cdot \sigma\right|^{2} d s=O(1)
$$

which replaces (3.12). We conclude that (instead of (3.13)

$$
E\left|\int_{0}^{t} g_{1}(\xi(s)) d s\right|^{2} \leq C .
$$

Let $0<r_{0}<r_{1}$ and let $\bar{g}_{2}(r)$ be a continuous function satisfying $\bar{g}_{2}(r)=$ $\epsilon /(1+r)^{1+\delta}$ if $r>r_{0}, \bar{g}_{2}(r)=0$ if $r<r_{0} / 2$. Take

$$
F_{0}(r)=C \int_{0}^{r} e^{-I(s)} \int_{0}^{r} e^{I(\tau)} \bar{g}_{2}(\tau) d \tau \quad(C>0) .
$$

Since $\delta>1$,

$$
F_{0}(r) \leq C, \quad F_{0}^{\prime}(r) \leq C / r^{k} \quad\left(k=\min \left(\delta, 3^{\prime} / 2\right)\right)
$$

instead of (3.14). It follows that

$$
E\left|\int_{0}^{t} g_{2}(|\xi(s)|) d s\right|^{2} \leq C+2 E \int_{0}^{t}\left|\nabla_{x} F_{0} \cdot \sigma\right|^{2} d s
$$

and by, Lemma 3(b) with $a=2 \delta, E \int_{0}^{t}\left|\nabla_{x_{0}} F_{0} \cdot \sigma\right|^{2} d s \leq C$. Thus, the left-hand side of (3.24) is bounded by a constant. Combining this with (3.22), (3.8) we obtain

Lemma 5. Let the conditions (2.2), (2.3), (2.32) bold, let $d \geq 3$ and let (2.16) bold with some $\delta>1$. Then

$$
E\left|\int_{0}^{t} a_{j}(s, \xi(s)) d s\right|^{2}=O(1) .
$$

Remark 1. If in Lemma 4 we replace the condition (2.16) by

$$
\sum_{i}\left|a_{i}(t, x)\right| \leq C /(1+|x|)^{1+\delta}
$$


where $0<\delta<1$, then

$$
E\left|\int_{0}^{t} a_{j}(s, \xi(s)) d s\right|^{2}=O\left(t^{1-\delta}\right) .
$$

Remark 2. If in Lemma 4 we replace (2.16) by (3.26) with $\delta=1$ and if either $d \geq 3$ or $d \geq 2$ and

$$
\sum_{i, j}\left|b_{i j}(t, x)-\bar{b}_{i j}\right| \leq C /(1+|x|)^{\beta} \text { for some } \beta>0,
$$

then, by the proofs of Lemmas 4, 5 and upon applying Lemma 3(c), we find that

$$
E\left|\int_{0}^{t} a_{j}(s, \xi(s)) d s\right|^{2}=O(\log t) .
$$

We shall need the following condition:

(3.30) $\sum_{i, j}\left|\sigma_{i j}(t, x)-\bar{\sigma}_{i j}\right| \leq \epsilon(|x|) /(1+|x|)^{\delta}, \quad \delta \geq 0, \epsilon(r) \rightarrow 0$ if $r \rightarrow \infty$, where $\bar{\sigma}_{i j}$ are constants. Note that (3.30) implies (2.35) with the same $\delta$ (but not the same $\epsilon(r)$ ) and that

$$
\bar{b}=1 / 2 \bar{\sigma} \bar{\sigma}^{*}, \quad 2 \operatorname{tr} \bar{b}=|\bar{\sigma}|^{2}
$$

We shall also need the condition

$$
\sum_{i, j}\left|\sigma_{i j}(t, x)-\bar{\sigma}_{i j}\right| \leq C /(1+|x|)^{\delta}, \quad \delta>0 .
$$

This condition implies (3.28) with $\beta=\delta$.

We can now state the main results on asymptotic estimates.

Theorem 5. (a) Let (2.2), (2.3), (2.16), (3.30) bold with $0 \leq \delta<1$, and let $d \geq 2$. Then

$$
E|\xi(t)-\bar{\sigma} w(t)|^{2}=o\left(t^{1-\delta}\right) .
$$

If (2.16), (3.30) are replaced by (3.26), (3.31) and $0<\delta<1$, then

$$
E|\xi(t)-\bar{\sigma} w(t)|^{2}=O\left(t^{1-\delta}\right)
$$

(b) Let (2.2), (2.3), (2.16), (3.30) bold with $\delta=1$ and let $d \geq 3$. Then

$$
E|\xi(t)-\bar{\sigma} w(t)|^{2}=o(\log t) .
$$

If (2.16), (3.30) are replaced by (3.26), (3.31) and $\delta=1$, then

$$
E|\xi(t)-\bar{\sigma} w(t)|^{2}=O(\log t) .
$$

(c) Let (2.2), (2.3), (3.26), (3.31) bold for some $\delta>1$ and let $d \geq 3$. Then

$$
E|\xi(t)-\bar{\sigma} w(t)|^{2}=O(1) .
$$

Proof. Let the conditions (2.2), (2.3), (2.16), (3.30), $0 \leq \delta<1, d \geq 2$, of (a) hold. Consider 


$$
E\left|\int_{0}^{t}(\sigma-\bar{\sigma}) d w(s)\right|^{2}=E \int_{0}^{t}|\sigma-\bar{\sigma}|^{2} d s .
$$

Since $|\sigma(t, x)-\bar{\sigma}|^{2} \leq \epsilon^{2}(|x|) /(1+|x|)^{2 \delta}$, Lemma 3(a) implies that

$$
E \int_{0}^{t}|\sigma(s, \xi(s))-\bar{\sigma}|^{2} d s=o\left(t^{1-\delta}\right) .
$$

Hence

Writing

$$
E\left|\int_{0}^{t}(\sigma(s, \xi(s))-\bar{\sigma}) d w(s)\right|^{2}=o\left(t^{1-\delta}\right)
$$

$$
\xi(t)-\bar{\sigma} w(t)=\xi_{0}+\int_{0}^{t} a(s, \xi(s)) d s+\int_{0}^{t}[\sigma(s, \xi(s))-\bar{\sigma}] d w(s)
$$

and using (3.37) and Lemma 4, the assertion (3.32) follows.

The proofs of (3.33)-(3.36) are similar. In proving (3.33)-(3.35) we use Remarks 1, 2 following Lemma 5, and in the proof of (3.36) we employ Lemma 5.

Remark. In all the results of $\$ \S 2,3$ we have assumed the condition (2.3). By slight changes in the proofs one can verify that if

$$
(1+|x|) \sum_{i}\left|a_{i}(t, x)\right| \leq \epsilon \quad \text { for all } t \geq 0, x \in R^{m}
$$

where $\epsilon$ is sufficiently small, then the condition (2.3) may be omitted.

4. Applications of the asymptotic estimates. For convenience we restate some of the assumptions made in Theorem 5.

$$
\lim _{|x| \rightarrow \infty} \sigma_{i j}(t, x)=\bar{\sigma}_{i j} \quad \text { uniformly with respect to } t, 1 \leq i, j \leq m,
$$

$$
\lim _{|x| \rightarrow \infty}(1+|x|) \sum_{i}\left|a_{i}(t, x)\right|=0 \quad \text { uniformly with respect to } t \text {. }
$$

Theorem 5(a) with $\delta=0$ states

If (2.2), (2.3), (4.1), (4.2) hold and if $d \geq 2$ then

$$
E|\xi(t)-\bar{\sigma} w(t)|^{2}=o(t)
$$

From (4.3) if follows that $\xi(t) / \sqrt{t}-\bar{\sigma} w(t) / \sqrt{t} \rightarrow 0$ in $L^{2}$; consequently also in probability. This immediately yields the following theorem on convergence in distribution of $\xi(t)$ :

Theorem 6. Let the assumptions (2.2), (2.3), (4.1), (4.2) bold, let $\bar{\sigma}$ be nonsingular matrix, and let $m \geq 2$. Then

$$
\lim _{t \rightarrow \infty} P\{\xi(t)<x \sqrt{t}\}
$$

$$
=\frac{1}{(2 \pi)^{m / 2} \operatorname{det} \bar{\sigma}} \int_{-\infty}^{x_{1}} \ldots \int_{-\infty}^{x_{m}} \exp \left\{-\frac{1}{2} \sum_{i, j=1}^{m} \hat{b}_{i j} y_{i} y_{j}\right\} d y_{1} \cdots d y_{m}
$$


where $\hat{b}$ is the inverse matrix to $\bar{b}$.

Suppose next that (2.2), (2.3) hold and that

$$
\begin{gathered}
\sum_{i, j}\left|\sigma_{i j}(t, x)-\bar{\sigma}_{i j}\right| \leq C /(1+|x|)^{\delta}, \\
\sum_{i}\left|a_{i}(t, x)\right| \leq C /(1+|x|)^{1+\delta}
\end{gathered}
$$

for some $0<\delta<1$. Suppose $\bar{\sigma}$ is nonsingular, $m \geq 2$, and denote by $\hat{\sigma}$ the inverse of $\bar{\sigma}$. Then

$$
\frac{\hat{\sigma} \xi(t)-w(t)}{\sqrt{2 t \log \log t}}=\frac{\hat{\sigma}}{\sqrt{2 t \log \log t}} \int_{0}^{t} a(s, \xi(s)) d s
$$

$$
\begin{aligned}
&+\frac{\hat{\sigma}}{\sqrt{2 t \log \log t}} \int_{0}^{t}[\sigma(s, \xi(s))-\bar{\sigma}] d w(s)+\frac{\hat{\sigma} \xi_{0}}{\sqrt{2 t \log \log t}} . \\
& \equiv J_{1}(t)+J_{2}(t)+J_{3}(t) \equiv J(t) .
\end{aligned}
$$

We shall denote various positive constants by the same symbol $C$. By the proof of Theorem 5(a), if $t_{n}=n^{\lambda}, \lambda=4 / \delta, n$ large,

$$
\begin{aligned}
& P\left\{\sup _{t} \leq t \leq \leq_{n+1}(t) \mid>\frac{1}{n}\right\} \leq P\left\{\frac{C}{\sqrt{t_{n}} \cdot 0^{\cdot t}+1}|a(s, \xi(s))| d s>\frac{1}{n}\right\} \\
& \leq \frac{C n^{2}}{t_{n}} E\left|\int_{0}^{t}{ }_{n+1}\right| a(s, \xi(s))|d s|^{2} \leq \frac{{ }^{\prime} C n^{2}}{t_{n}}\left(t_{n+1}\right)^{1-\delta} \leq \frac{C}{n^{2}} .
\end{aligned}
$$

Next, by the matringale inequality and the proof of Theorem 5(a),

$$
\begin{aligned}
P\left\{\sup _{t_{n} \leq t \leq t_{n+1}}\left|J_{2}(t)\right|>\frac{1}{n}\right\} & \\
& \leq P\left\{\frac{C}{\sqrt{t_{n}}} \sup _{t_{n} \leq t \leq t_{n+1}}\left|\int_{0}^{t}[\sigma(s, \xi(s))-\bar{\sigma}] d w(s)\right|>\frac{1}{n}\right\} \\
& \leq \frac{C n^{2}}{t_{n}}\left(t_{n+1}\right)^{1-\delta} \leq \frac{C}{n^{2}} .
\end{aligned}
$$

Since, finally,

$$
P\left\{\sup _{t_{n} \leq t \leq t_{n+1}}\left|J_{3}(t)\right|>\frac{1}{n}\right\} \leq \frac{C n^{2}}{t_{n}} E\left|\xi_{0}\right|^{2} \leq \frac{C}{n^{2}},
$$

we conclude that

$$
P\left\{\sup _{t{ }_{n} \leq t \leq t_{n+1}}|J(t)|>\frac{1}{n}\right\}<\frac{1}{n^{2}} .
$$


Hence, by the Borel-Cantelli lemma,

$$
P\left\{\sup _{t_{n} \leq t \leq t_{n+1}}|J(t)|>\frac{1}{n} \quad \text { i.o. }\right\}=0 ;
$$

consequently

$$
P\left\{\lim _{t \rightarrow \infty}|J(t)|=0\right\}=1 .
$$

Recalling the law of the iterated logarithm for Brownian motion (see [1], [2]) we obtain from (4.7) the following law:

Theorem 7. Let the conditions (2.2), (2.3), (4.5), (4.6) bold for some $\delta>0$, let $\bar{\sigma}$ be nonsingular, and let $m \geq 2$. Then, for any $i, 1 \leq i \leq m$,

$$
\begin{aligned}
& P\left\{\varlimsup_{t \rightarrow \infty} \frac{\sum_{j=1}^{m} \hat{\sigma}_{i j} \xi_{j}(t)}{\sqrt{2 t \log \log t}}=1\right\}=1, \\
& P\left\{\prod_{t \rightarrow \infty}^{\lim } \frac{\sum_{j=1}^{m} \hat{\sigma}_{i j} \xi_{j}(t)}{\sqrt{2 t \log \log t}}=-1\right\}=1 .
\end{aligned}
$$

Similarly one can prove that

$$
P\left\{\varlimsup_{t \rightarrow \infty} \frac{|\hat{\sigma} \xi(t)|}{\sqrt{2 t \log \log t}}=1\right\}=1 .
$$

It was proved by Dvoretsky and Erdös [3] (see also [11]) that if $m \geq 3$ then

$$
P\left\{\lim \left(|w(t)| / t^{\alpha / 2}\right)=\infty\right\}=1 \text { for any } 0<\alpha<1 .
$$

Clearly this holds also for $\bar{\sigma} w(t)$ if $d \geq 3$. But under the assumptions of Theorem 7 (with $\alpha>1-\delta$ ) we get (cf. the proof of (4.8))

Hence,

$$
P\left\{\lim _{t \rightarrow \infty} \frac{|\xi(t)-\bar{\sigma} w(t)|}{t^{a / 2}}=0\right\}=1 .
$$

Theorem 8. Let the assumptions (2.2), (2.3), (4.5), (4.6) bold for some $\delta>0$ and let $d \geq 3$. Then

$$
P\left\{\lim _{t \rightarrow \infty} \frac{|\xi(t)|}{t^{\alpha / 2}}=\infty\right\}=1 \text { for any } 0<a<1 .
$$

Our last application is to the Cauchy problem for parabolic equations with time independent coefficients. Under some standard conditions (see [4]) there exists a unique solution of the Cauchy problem 


$$
L u=\sum_{i, j=1}^{m} b_{i j}(x) \frac{\partial^{2} u}{\partial x_{i} \partial x_{j}}+\sum_{i=1}^{m} a_{i}(x) \frac{\partial u}{\partial x_{i}}-\frac{\partial u}{\partial t}=0 \quad \text { for } 0<t<\infty, x \in R^{m},
$$

$$
u(0, x)=f(x) \text { for } x \in R^{m}
$$

and it can be written in the form (see [5], [6])

$$
u(t, x)=E f\left(\xi_{x}(t)\right)
$$

where $\xi_{x}(t)$ is the solution of $(1.1)$ with $\xi(0)=x$.

These standard conditions are satisfied if

(i) $a_{i}(x), \sigma_{i j}(x)$ are bounded functions in $R^{m}$, locally Lipschitz continuous, uniformly Hölder continuous, and $\left(b_{i j}(x)\right)$ is uniformly positive definite.

(ii) $f(x)=O(|x|)$ as $|x| \rightarrow \infty$ and $f$ is locally Hölder continuous.

The uniqueness of the solution is within the class of functions $z(t, x)$ satisfying, for each $T>0$,

$$
|z(t, x)| \leq C \exp \left\{\beta|x|^{2}\right\} \quad\left(0 \leq t \leq T, x \in R^{m}\right)
$$

where $C, \beta$ are positive constants, depending on $T$.

Let us further assume that

$$
\begin{aligned}
\sum_{i, j}\left|\sigma_{i j}(x)-\delta_{i j}\right| & =O\left(|x|^{-\delta}\right) \quad \text { as }|x| \rightarrow \infty, \\
\sum_{i}\left|a_{i}(x)\right| & =O\left(|x|^{-1-\delta}\right) \text { as }|x| \rightarrow \infty,
\end{aligned}
$$

and replaced (ii) by the stronger condition

$$
|f(x)-f(y)| \leq A|x-y| \quad(A \text { constant }) .
$$

If $b_{i j}=1 / 2 \delta_{i j}, a_{i}=0$, then the solution $v(t, x)$ of (4.12), (4.13) is given by $E f(w(t)+x)$, i.e.

$$
v(t, x)=\frac{1}{(2 \pi)^{m / 2} t^{m / 2}} \int_{R^{m}} \exp \left\{\frac{|x-\xi|^{2}}{2 t}\right\} f(\xi) d \xi .
$$

Hence, by (4.14), (4.17),

$$
|u(t, x)-v(t, x)| \leq C\left\{E\left|\xi_{x}(t)-w(t)\right|^{2}\right\}^{1 / 2}+C .
$$

We can now apply Theorems $5(\mathrm{a}), 5(\mathrm{c})$ to estimate the right-hand side. A careful review of the proof of these theorems shows how the estimates depend on the parameter $x$. This leads to the following theorem.

Theorem 9. Let the conditions (i), (4.15), (4.16), (4.17) bold. If $m \geq 2$, $0<\delta<1$, then, for all $t \geq 0, x \in R^{m}$,

$$
|u(t, x)-v(t, x)| \leq C\left[t^{(1-\delta) / 2}+(1+|x|)^{1-\delta}+1\right] \quad(C \text { constant }) .
$$


If $m \geq 3$ and $\delta>1$ then, for all $t \geq 0, x \in R^{m}$,

$$
|u(t, x)-v(t, x)| \leq C \quad(C \text { constant }) .
$$

5. The case $m=1$. In proving Theorem 5(a), or even in deriving (4.3), we have assumed that the matrix $\bar{b}$ has at least two positive eigenvalues. This assumption is essential. To see what may happen when $\bar{b}$ has only one positive eigenvalue, we resort to the case $m=1$ and, for simplicity, take an equation of the form

$$
d \xi(t)=a(\xi(t)) d t+d w(t)
$$

We assume that $a^{\prime}(x)$ is continuous and

$$
\int_{-\infty}^{\infty}|a(x)| d x<\infty .
$$

One can construct in the present case comparison functions by directly solving equations of the form

$$
L f=1 / 2 f^{\prime \prime}(x)+a(x) f^{\prime}(x)=\psi(x) .
$$

Setting $A(x)=2 \int_{-\infty}^{x} a(y) d y$, the general solution of (5.3) is given by

$$
f(x)=\int_{0}^{x} e^{-A(z)} d z\left[2 \int_{0}^{z} e^{A(y)} \psi(y) d y+C_{1}\right]+C_{2}
$$

where $C_{1}, C_{2}$ are constants. Using this solution in the proof of Theorem 5 , we can derive the estimate

$$
E|\xi(t)-w(t)|^{2}=o(t)
$$

provided $A(-\infty)=0$, i.e.

If further

$$
\int_{-\infty}^{\infty} a(x) d x=0
$$

$$
|a(x)| \leq C /(1+|x|)^{1+\delta} \quad(0<\delta<1)
$$

then

$$
E|\xi(t)-w(t)|^{2}=O\left(t^{1-\delta}\right) .
$$

If $(5.5)$ is not satisfied then (5.4) is false. In fact, as proved by Kulinic [7], if $(5.5)$ is not satisfied (where $a(x)$ is any continuous function satisfying (5.2)) then already the assertion of Theorem 6 is invalid for any solution $\xi(t)$.

There is an intuitive reason why for $m=1, \sigma \equiv 1$ the assertion of Theorem 6 cannot hold unless $(5.5)$ is satisfied. In order for the distribution of $\xi(t) / \sqrt{t}$ to approximate the normal distribution as $t \rightarrow \infty$ the particles represented by $\xi(t)$, or $\xi(t, \omega)$, must be able to move without "resistance" from intervals $(\alpha, \beta)$ near $+\infty$ to intervals $(-\beta,-\alpha)$. Since in performing this move they must cross the interval $(-\alpha, \alpha)$, they are subject to the influence of the drift term $a(x)$. 
This drift coefficient will resist the movement if $\int_{-\infty}^{\infty} a(x) d x>0$; thus this inequality cannot take place. Similarly, the reverse inequality cannot take place.

If $m \geq 2$ and $\bar{b}$ is nonsingular then $\xi(t)$ may move from one $m$-dimensional interval in a neighborhood $G$ of infinity to another without leaving $G$. If the drift coefficient $a(t, x)$ is "small" in $G$ (i.e., if $|a(t, x)| \leq C(1+|x|)^{-1-\mu}, \mu>0$ ) then there will be negligible resistance by the drift coefficient to the movement of $\xi(t)$ in $G$. Thus, no condition analogous to (5.5) is required in the case $m \geq 2$.

So far we have considered only the case $\sigma=1$. The case of general $\sigma(x)>0$ can be reduced to the previous case by a simple transformation. Setting $\eta(t)=$ $f(\xi(t)), f(z)=\int_{0}^{z} d y / \sigma(y)$, the equation

$$
d \xi(t)=a(\xi(t)) d t+\sigma(\xi(t)) d w(t)
$$

reduces to

$$
d \eta=\left[a(g(\eta(t))) / \sigma(g(\eta(t)))-1 / 2 \sigma^{\prime}(g(\eta(t)))\right] d t+d w(t)
$$

where $g$ is the inverse function of $f$. The condition (5.5) for the equation (5.9) becomes

$$
\int_{-\infty}^{\infty} \frac{a(z)-1 / 2 \sigma(z) \sigma^{\prime}(z)}{\sigma^{2}(z)} d z=0 .
$$

If

$$
\left|a(g(y)) / \sigma(g(y))-1 / 2 \sigma^{\prime}(g(y))\right| \leq C /(1+|y|)^{1+\delta} \quad(\delta>0)
$$

and if (5.10) holds, then (by the assertion (5.7) applied to the equation (5.9))

Assuming also that

$$
E|\eta(t)-w(t)|^{2} \leq C t^{1-\delta}+C
$$

$$
|\bar{\sigma} f(x)-x| \leq C\left(|x|^{1-\mu}+1\right) \quad(0 \leq \mu<1, \bar{\sigma}>0)
$$

for some constant $\bar{\sigma}$, we easily derive

$$
E|\xi(t)-\bar{\sigma} w(t)|^{2} \leq K t^{1-\nu}+K^{\prime} \quad \text { for all } t \geq 0,
$$

where $\nu=\min (\delta, \mu)$ and $K, K^{\prime}$ are positive constants.

Noting that if

$$
|\sigma(x)-\bar{\sigma}| \leq C /(1+|x|)^{\mu}
$$

then (5.12) holds, and that (5.11) is equivalent to

$$
\left|a(x) / \sigma(x)-1 / 2 \sigma^{\prime}(x)\right| \leq C /(1+|x|)^{1+\delta}
$$

(provided (5.14) holds), we can state

If (5.15), (5.14) and (5.10) bold, then for any solution $\xi(t)$ of $(5.8)(m=1)$ the estimate (5.13) bolds.

If $(5.14)$ is replaced by 


$$
\bar{\sigma}=\lim _{|x| \rightarrow \infty} \sigma(x) \text { exists, } \bar{\sigma}>0
$$

if the left-hand side of $(5.11)$ is assumed to be absolutely integrable over $(-\infty, \infty)$, and if (5.10) holds, then one can show that

$$
E\left|\xi(t)-\bar{\sigma} u^{\prime}(t)\right|^{2}=o(t) \text {. }
$$

Except for Theorem 7, the applications given in $\$ 4$ extend to the case $m=1$.

6. Counterexample. We shall give an example of a system of $m$ equations $(m \geq 1)$

$$
d \xi(t)=a(\xi(t)) d t+d w(t)
$$

for which

$$
a(x)=O\left(\frac{\log |x|}{|x|}\right) \text { if }|x| \rightarrow \infty,
$$

such that the estimate

$$
E|\xi(t)|^{2} \leq K t+K^{\prime} \quad \text { for all } t \geq 0\left(K, K^{\prime} \text { positive constants }\right)
$$

is false.

Let $f(x)$ be a $C^{2}\left(R^{m}\right)$ function such that

$$
f(x)=r^{2} / \log r \text { if } r=|x|>2 .
$$

If $a_{i}(x)=x_{i} \alpha(r)$ for $|x|>2$, then

$$
\begin{aligned}
\frac{1}{2} \Delta f(x) & +\sum_{i=1}^{m} a_{i}(x) \frac{\partial f(x)}{\partial x_{i}} \\
& =\frac{1}{\log r}\left[1-\frac{m+2}{2} \frac{1}{\log r}+\frac{1}{(\log r)^{2}}\right]+\frac{2 r^{2}}{\log r}\left[1-\frac{1}{2 \log r}\right] \alpha(r) .
\end{aligned}
$$

Let

$$
\alpha(r)=\frac{\log r}{2 r^{2}}[1+\beta(r)]
$$

and define $\beta(r)$ so that

$$
\frac{1}{2} \Delta f(x)+\sum_{i=1}^{m} a_{i}(x) \frac{\partial f(x)}{\partial x_{i}}=1 \quad \text { if }|x|>2 .
$$

It is easily seen that $\beta(r)=O(1)$. We now define $a_{i}(x)$ in $R^{m}$ as a continuous function such that

$$
a_{i}(x)=x_{i}[\log |x|+\beta(|x|)] / 2|x|^{2} \quad \text { if }|x|>2 .
$$

By Ito's formula and (6.5),

$$
E f(\xi(t))=E f\left(\xi_{0}\right)+E \int_{0}^{1} c(\xi(s)) d s
$$

where $c(x)-1=0$ if $|x|>2$. 
We wish to a pply the proof of Lemma 1 to $\phi(t, x)=c(x)-1$. Here the $a_{i}(t, x)=a_{i}(x)$ are not bounded by $\epsilon(|x|) /(1+|x|)$ where $\epsilon(r) \rightarrow 0$ if $r \rightarrow \infty$. Nevertheless (2.11) takes the form

$$
\frac{1}{2}[1+\theta(r)] \leq \frac{1}{2} m+\sum_{i}\left(x_{i}-e_{i}\right) a_{i}(x)
$$

where $r=|x-e|$ and $e=\left(e_{1}, \cdots, e_{m}\right)$ is a point not contained in the support of $c(x)-1$, i.e. $|e|>2$. In view of (6.6) and the fact that $\beta(r)=O(1)$, we can construct $\theta(r)$ satisfying (6.8) such that, for any $A>0, \lim _{r \rightarrow \infty} \theta(r)=A$. Hence, by the proof of Lemma 1,

$$
E\left|\int_{0}^{t}[c(\xi(s))-1] d s\right|=O(1) \text { as } t \rightarrow \infty .
$$

Combining (6.9) with (6.7) we get

$$
|E f(\xi(t))-t| \leq C_{0} \quad \text { for } t \geq 0 \quad\left(C_{0} \text { constant }\right) .
$$

For any $\epsilon>0$ there is a $B>0$ such that

$$
|x|^{2} / \log |x| \leq \epsilon|x|^{2}+B \text { if }|x|>2 .
$$

Hence

$$
f(x) \leq \epsilon|x|^{2}+C \text { for all } x \in R^{m}
$$

where $C$ is a constant depending on $\epsilon$. This implies

$$
E f(\xi(t)) \leq \epsilon E|\xi(t)|^{2}+C .
$$

Now, if (6.3) holds then from (6.10), (6.11) we obtain

$$
t \leq \epsilon K t+\epsilon K^{\prime}+C+C_{0} \text { for all } t \geq 0 .
$$

But this is impossible if $\epsilon<1 / K$.

If the function $\log |x|$ occurring in (6.2) is replaced by other functions which increase to infinity as $|x| \rightarrow \infty$, such that $\log \log |x|$, then we can again show by the above method that (6.3) cannot hold. If, however, $a(x)=O\{1 /|x|\}$, then (6.3) holds (by Theorem 2). Recall that if (3.2) holds then (3.1) is also valid.

7. Equations with unbounded coefficients. We shall extend some of the results of $\$ \$ 2,3$ to equations with unbounded coefficients.

Theorem 10. Assume that

$$
\sum_{i} b_{i i}(t, x) \leq C\left(1+|x|^{2}\right)^{\mu}, \quad\left|\sum x_{i} a_{i}(t, x)\right| \leq C\left(1+|x|^{2}\right)^{\mu}
$$

for some constants $C>0,0 \leq \mu<1$. Then

$$
E|\xi(t)|^{2-2 \mu} \leq K t+K^{\prime} \quad \text { for all } t \geq 0
$$

where $K, K^{\prime}$ are positive constants. 
Proof. Set $\phi(x)=\left(1+|x|^{2}\right)^{1-\mu}$. Then $L \phi \leq C$ where $C$ is a constant.

Using Ito's formula we find that

$$
E\left(1+|\xi(t)|^{2}\right)^{1-\mu} \leq C t+C
$$

this yields (7.1).

To get a lower bound on $E|\xi(t)|^{2-2 \mu}$, we set

$$
\begin{gathered}
a_{i}(t, x)=\widetilde{a}_{i}(t, x)\left(1+|x|^{2}\right)^{\mu}, \\
\sigma_{i j}(t, x)=\widetilde{\sigma}_{i j}(t, x)\left(1+|x|^{2}\right)^{\mu / 2} .
\end{gathered}
$$

Then $\tilde{b}(t, x)=1 / 2 \tilde{\sigma}(t, x) \tilde{\sigma} *(t, x)$ satisfies

$$
b_{i j}(t, x)=\tilde{b}_{i j}(t, x)\left(1+|x|^{2}\right)^{\mu} .
$$

Lemma 6. Let the assumptions made in Lemma 1 be satisfied for $a_{i}, b_{i j}$ replaced by $\tilde{a}_{i}, \tilde{b}_{i j}$. Then for any bounded measurable function $\phi(x)$ with compact support

$$
E\left|\int_{0}^{t} \phi(\xi(s)) d s\right| \leq K_{1}+K_{2} t^{(1+\eta) /(2-2 \mu)} \quad(t \geq 0)
$$

where $K_{1}, K_{2}$ are constants and $\eta$ is as in Lemma 1 .

Proof. The proof is similar to that of Lemma 1. The only difference is that we introduce $\psi$ such that $|\phi(x)| \leq\left(1+|x|^{2}\right)^{\mu} \psi(|x|)$, and then construct $f(x)=$ $F(r)$ satisfying $L f(x) \geq\left(1+|x|^{2}\right)^{\mu} \psi(|x|)$. We obtain

$$
E\left|\int_{0}^{t} \phi(\xi(s)) d s\right| \leq C E|\xi(t)|^{1+\eta}
$$

and then use Theorem 10.

If $\phi(x)$ does not have a compact support, but

$$
|\phi(x)| \leq C\left(1+|x|^{2}\right)^{\mu} /(1+|x|)^{\alpha},
$$

then we can proceed as in Lemma 2 . We find that

$$
E\left|\int_{0}^{t} \phi(\xi(s)) d s\right| \leq C t^{(1+\eta) /(2-2 \mu)}+C t^{(2-\alpha) /(2-2 \mu)} \text {. }
$$

Theorem 11. Let (2.2)-(2.5) bold with $a_{i}, b_{i j}$ replaced by $\tilde{a}_{i}, \widetilde{b}_{i j}$, and let $0 \leq \mu<1$. Then

$$
E|\xi(t)|^{2-2 \mu} \geq K t-K^{\prime} \quad \text { for all } t \geq 0
$$

where $K, K^{\prime}$ are positive constants.

Proof. Note that

$$
L\left(1+|x|^{2}\right)^{\mu} \geq \gamma-\phi(x)
$$

where $\gamma>0$ and $\phi(x)$ has compact support, and apply Lemma 6 .

We finally note that using the result preceding Theorem 11 one can proceed to 
establish an analog of Theorem 5. A typical estimate has the form

$$
E\left|\xi(t)-\bar{\sigma} \int_{0}^{t}\left[1+|\xi(s)|^{2}\right]^{\mu} d w(s)\right|^{2}=O\left(t^{2 /(2-2 \mu)-\epsilon}\right)
$$

for some $\epsilon>0$, where $\bar{\sigma}=\lim |x| \rightarrow \infty \tilde{\sigma}(t, x)$. The estimate (7.7) does not appear to be interesting when $\mu>0$.

The case $\mu<0$ can be treated in a similar fashion. The assertion (7.1) remains true for $\mu<0$ provided

$$
\sum_{i, j}\left|b_{i j}(t, x)\right|+\left|\sum_{i} x_{i} a_{i}(t, x)\right| \leq C\left(1+|x|^{2}\right)^{\mu},
$$

and the assertion (7.6) remains true under the same assumptions as in Theorem 11.

\section{REFERENCES}

1. L. Breiman, Probability, Addison-Wesley, Reading, Mass., 1968. MR 37 \#4841.

2. J. L. Doob, Stochastic processes, Wiley, New York, 1953. MR 15, 445.

3. A. Dvoretsky and P. Erdös, Some problems on random walk in space, Proc. Second Berkeley Sympos. on Math. Statist. and Probability 1950, Univ. of California Press, Berkeley, Calif., 1951, pp. 353-367. MR 13, 852.

4. A. Friedman, Partial differential equations of parabolic type, Prentice-Hall, Englewood Cliffs, N. J., 1964. MR 31 \#6062.

5. I. I. Gihman and A. V. Skorohod, Introduction to the theory of random processes, "Nauka", Moscow, 1965; English transl., Saunders, Philadelphia, Pa., 1969. MR 33 \#6689; MR 40 \#923.

6. - Stochastic differential equations, "Naukova Dumka", Kiev, 1968. (Russian) MR $41 \# 7777$.

7. G. L. Kulinič, On the limit behavior of the distribution of the solution of a stochastic diffusion equation, Teor. Verojatnost. i Primenen. 12 (1967), 548-551 = Theor. Probability Appl. 12 (1967), 497-499. MR 35 \#6206.

8. L Limiting behavior of distributions of a solution of the stochastic diffusion equation, Ukrain. Mat. Ž. 19 (1967), no. 2, 119-125. (Russian) MR 35 \#2347.

9. - A symptotic no rmality of the distribution of the solution of a stochastic diffusion equation, Ukrain. Mat. Ž. 20 (1968), 396-400. (Russian) MR 38 \#766.

10. - Limit distributions of a solution of the stochastic diffusion equation, Teor. Verojatnost. i Primenen. 13 (1968), 502-506 = Theor. Probability Appl. 13 (1968), 478-482. MR $39 \# 1028$.

11. P. Levy, Le mouvement Brownien, Mémor. Sci. Math., no. 126, Gauthier-Villars, Paris, 1954. MR 16, 601 .

12. D. W. Stroock and S. R. S. Varadhan, Diffusion processes with continuous coefficients. I, II, Comm. Pure Appl. Math 22 (1969), 345-400, 479-530. MR $40 \# 6641$; \#8130.

DEPARTMENT OF MAT HEMATICS, NORTHWESTERN UNIVERSITY, EVANSTON, ILLINOIS 60201 (Current address)

DEPAR TMENT OF MATHEMATICS, TEL AVIV UNIVERSITY, TEL AVIV, ISRAEL 